\title{
Comparison of daily rhythms of oxygen metabolites and serum barrier to oxidation in domestic animals
}

Research Article

\author{
Department of Experimental Sciences and Applied Biotechnology, \\ Laboratory of Veterinary Chronophysiology, \\ Faculty of Veterinary Medicine, University of Messina, \\ 98168 Messina, Italy
}

Claudia Giannetto, Francesco Fazio, Stefania Casella, Anna Assenza, Simona Marafioti, Giuseppe Piccione*

Received 26 July 2010; Accepted 30 September 2010

\begin{abstract}
The aim of this study was to compare the daily rhythm of reactive oxygen metabolites (hydroperoxides), and serum barrier to oxidation in five different mammal species. Five female Comisana sheep, five female Maltese goats, five female Bruna cows and five female Thoroughbred horses were housed separately in boxes equipped with an opening window and five female Blue Vienna rabbits were individually housed in metallic cages. All animals were kept under natural photoperiod and indoor environmental conditions. Blood samples were collected every $3 \mathrm{~h}$ over a $48 \mathrm{~h}$ period for the assessment of hydroperoxides, antioxidant barrier (Oxy-ads) and thiol-antioxidant barrier (SHp). A two-way for repeated measures ANOVA showed a significant effect of time of day and different species on all studied parameters. Daily rhythmicity of hydroperoxides, Oxy-ads and SHp were observed in all studied species. Nocturnal acrophases were observed in sheep, goats and rabbits, diurnal acrophases were observed in cattle and horse. We conclude that there is a synergism of the daily rhythms of hydroperoxides and anti-oxidant power in the different species studied, and that this synergism is typical of each species.
\end{abstract}

Keywords: Daily rhythms • Hydroperoxides $\bullet$ Oxy-ads $\bullet$ SHp • Sheep • Goats • Cows • Horses $\bullet$ Rabbits

(C) Versita Sp. z 0.0 .

\section{Introduction}

In aerobic organisms, under physiological conditions, the use of oxygen by cells generates potentially deleterious reactive oxygen species (ROS) which include superoxide anion, hydroxyl radical and hydrogen peroxide $[1,2]$. As "witnesses" of the tissue oxidative damage, hydroperoxides are detectable in biological fluids by means of a reactive oxygen metabolite (hydroperoxides) test. In the plasma, a number of compounds are able to "buffer" the oxidant potential of reactive species of oxygen. Virtually, any donor of electrons is capable of opposing the oxidative attack of free radicals. Obviously, any injury to such "plasma/ serum barrier to oxidation" can result in oxidative tissue damage. The plasma/serum ability to oppose the massive oxidative action of a hypochlorous acid solution is evaluated by means of the oxy-adsorbent test (Oxy-ads), and a significant component of the plasma/serum barrier to oxidation is thiol. It is to oppose the propagation step of peroxidative processes by inactivating either alcohoxyl or hydroxyl radicals ( $\mathrm{SHp}$ test). The imbalance between oxidants and antioxidants at the cellular or individual level is commonly defined as "oxidative stress". In farm animals, oxidative stress may be involved in several pathological conditions, including conditions that are relevant for animal production and the general welfare of the individuals [3]. The utility of the hydroperoxides assay in monitoring oxidative stress in horses [4,5], sheep [6,7], goats [8], dogs [9] and dairy cows $[10,11]$ has been reported.

Gene expression that governs stress resistance behaviour, essential for maintaining a robust anti-oxidative defence, varies daily showing circadian rhythmicity in invertebrates, vertebrates and plants [12]. Rhythmic changes in oxidative damage of protein and lipid molecules are also reported [13]. In sheep and cows, circadian rhythm changes of oxidative stress markers in relation to reproductive status and daily total activity have been studied [14-16]. 
The aim of this study was to compare the daily rhythm of hydroperoxide production and serum barrier to oxidation in five domestic animals, specifically, in sheep, goats, cows, horses and rabbits.

\section{Experimental Procedures}

\subsection{Animals}

The experimental subjects were five female sheep (Comisana breed, 5 years old, mean body weight $47 \pm 3 \mathrm{~kg}$ ), five female goats (Maltese breed, 5 years old, mean body weight $44 \pm 2 \mathrm{~kg}$ ), five female cows (Bruna breed, 5 years old, mean body weight $600 \pm 50 \mathrm{~kg}$ ), five female horses (Thoroughbred, 4 years old, mean body weight $400 \pm 40 \mathrm{~kg}$ ) and five female rabbits (Blue Vienna Breed, 1 year old, mean body weight $4.5 \pm 0.4 \mathrm{~kg}$ ). All animals were clinically healthy and not pregnant. Animals were housed under natural photoperiod (sunrise 05:20; sunset 20:20) and indoor environmental conditions. Thermal and hygrometric records were collected inside the box for the whole study by means of a data logger (Gemini, UK). Minimal and maximal temperatures during the experimental period were $18^{\circ} \mathrm{C}$ and $22^{\circ} \mathrm{C}$, and mean humidity was $65-70 \%$. Sheep and goats were housed individually in $3 \times 3 \mathrm{~m}$ boxes equipped with an opening window. Cows and horses were housed individually in $5 \times 5 \mathrm{~m}$ boxes equipped with an opening window. Rabbits were individually housed in metallic cages $(90 \times 50 \times 35 \mathrm{~cm})$. Good-quality hay that was placed on the floor was available ad libitum. No feed supplements were provided to the animals. All animals had free access to water from a commercial watering place.

All treatments, housing and animal care reported above were carried out under the guidelines of the Italian Minister of Health for the care and use of animals (D.L. 27/1/1992, n 116) and UE (Directive 86/609/CEE).

\subsection{Blood sampling}

Blood samples were collected every $3 \mathrm{~h}$ over a $48 \mathrm{~h}$ period, starting at 08:00 on day 1 and finishing at 08:00 on day 3 . On each subject blood was collected from the jugular vein using vacutainer tubes with no anticoagulant. Blood samples were then centrifuged at $3000 \mathrm{~g}$ for $20 \mathrm{~min}$, and the obtained sera were immediately analyzed by means of a Slim spectrophotometer (Seac Florence, Italy), for the assessment of the following parameters: hydroperoxides, Oxy-ads and SHp. They were assessed with the so-called "spin traps" system, with molecules reacting with free radicals resulting in complexes visible with a spectrophotometer [17-19]. The hydroperoxide test assesses the concentration of hydroperoxides $(\mathrm{R}-\mathrm{OOH})$, a class of reactive metabolites of the oxygen in a biological sample (serum, plasma, tissues and cells). The Oxy-ads test assesses the anti-oxidant power of the plasmatic barrier by measuring its ability to contrast the oxidative action of hypochlorous acid. This acid is a highly oxidant compound that, in physiological conditions, is synthesized from activated polymorphonucleated leucocytes and acts as an oxidant against the attacks of the bacteria. The SHp test assesses the thiol anti-oxidant plasma barrier, which contrasts the propagation of the peroxidative processes by inactivating both the alkoxyl and the hydroxide radicals.

Dim red light (<3 lux, $15 \mathrm{~W}$ Safelight lamp filter 1A, Kodak Spa, Milan, Italy) was used for data collection during the dark phase.

\subsection{Statistical analysis}

A two-way for repeated measures analysis of variance (ANOVA) was applied to evaluate the effects of time of day and species on the parameters studied. AP value $<0.05$ was considered statistically significant.

A trigonometric statistical model was applied to each time series to describe the periodic phenomenon analytically, by characterizing the main rhythmic parameters according to the single cosinor procedure [20]. Four rhythmic parameters were determined: mesor, amplitude, acrophase and robustness. For each animal, the mesor of the rhythm was computed as the arithmetic mean of all values in the data set ( 9 data points per day). The amplitude of the rhythm was calculated as half the max-min range of the oscillation, which in turn was computed as the difference between peak and trough. Rhythm robustness was computed as a percentage of the maximal score attained by the chi-square periodogram statistic for ideal data sets of comparable size and 24-h periodicity [21]. Robustness greater than $40 \%$ is above noise level and indicates statistically significant rhythmicity.

\section{Results}

The application of a two-way ANOVA showed a statistically significant effect of time of day $\left(\mathrm{dRoms}: \mathrm{F}_{(16,320)}=6.37, \mathrm{P}<0.0001\right.$; Oxy-ads: $\mathrm{F}_{(16,320)}=4.37$, $\left.\mathrm{P}<0.0001 ; \quad \mathrm{SHp:} \quad \mathrm{F}_{(16,320)}=5.05, \quad \mathrm{P}<0.0001\right) \quad$ and different species (dRoms: $F(4,320)=3328, P<0.0001$; Oxy-ads: $\quad F(4,320)=508, \quad P<0.0001 ; \quad S H p$ : $F(4,320)=5173, P<0.0001)$ on all parameters studied. Serum hydroperoxide concentration was higher in rabbits $(P<0.001)$ than the other species studied, followed by horses $(P<0.001)$. The lowest serum 
hydroperoxide concentration was observed in goats $(P<0.001)$. Serum Oxy-ads concentration was lower in horses $(P<0.001)$ than in other species studied. No differences were observed between sheep and goats, they showed higher values $(P<0.001)$ than the other species. Serum SHp concentration was higher in horses
$(P<0.001)$ than in other species studied. No differences were observed between sheep and goats, they showed lower values $(P<0.001)$ than the other species. The application of the periodic model and the statistical analysis of the cosinor enabled us to define the periodic parameters during the $48 \mathrm{~h}$ of monitoring. Daily patterns

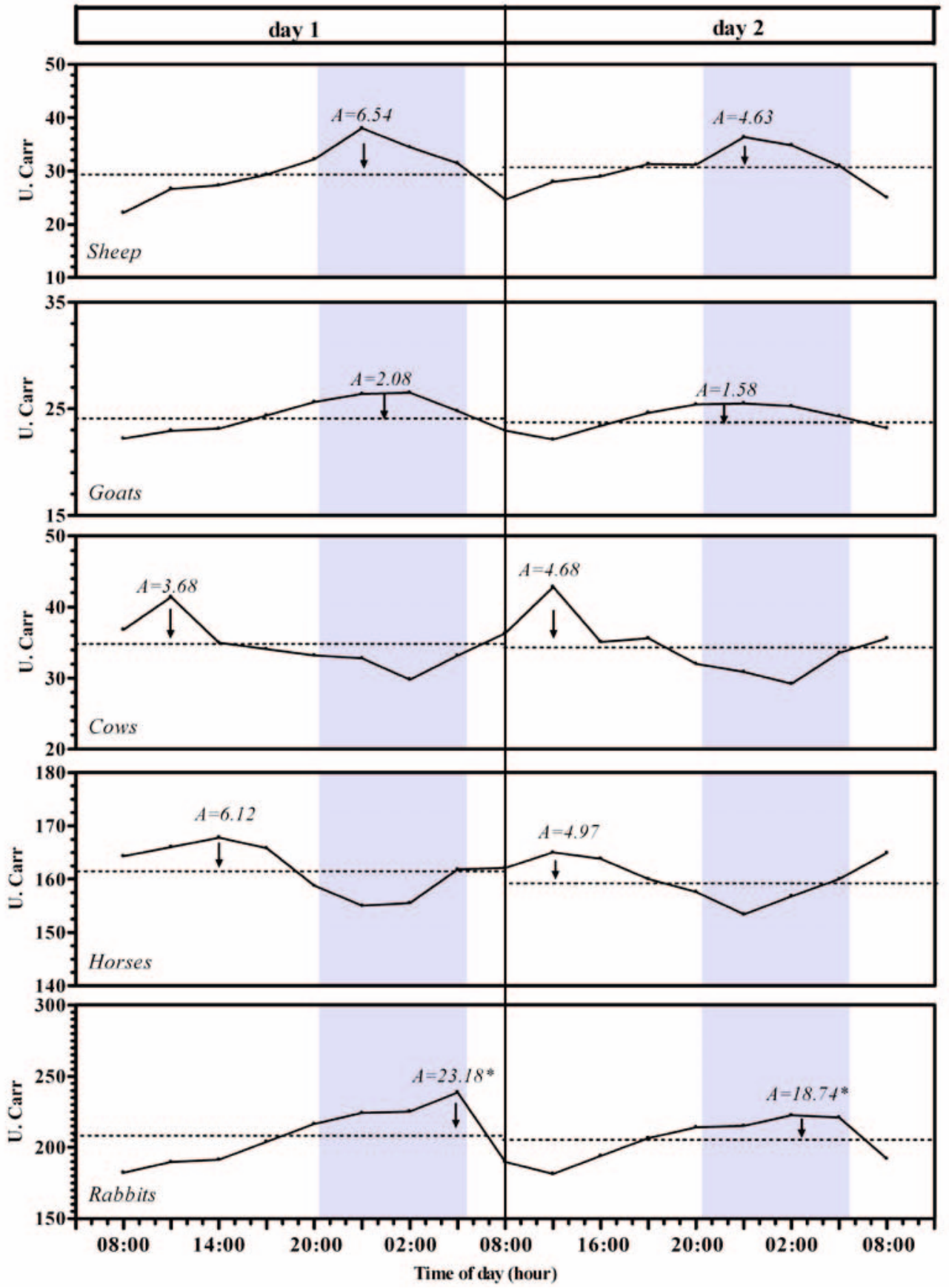

Figure 1. Daily patterns of hydroperoxides observed in sheep, goats, cows, horses and rabbits during the 48 hours of monitoring. Grey bars indicate the dark phase of photoperiod. Broken line indicates mesor value. A indicates amplitude value.

* Statistically significant as compared with other species 


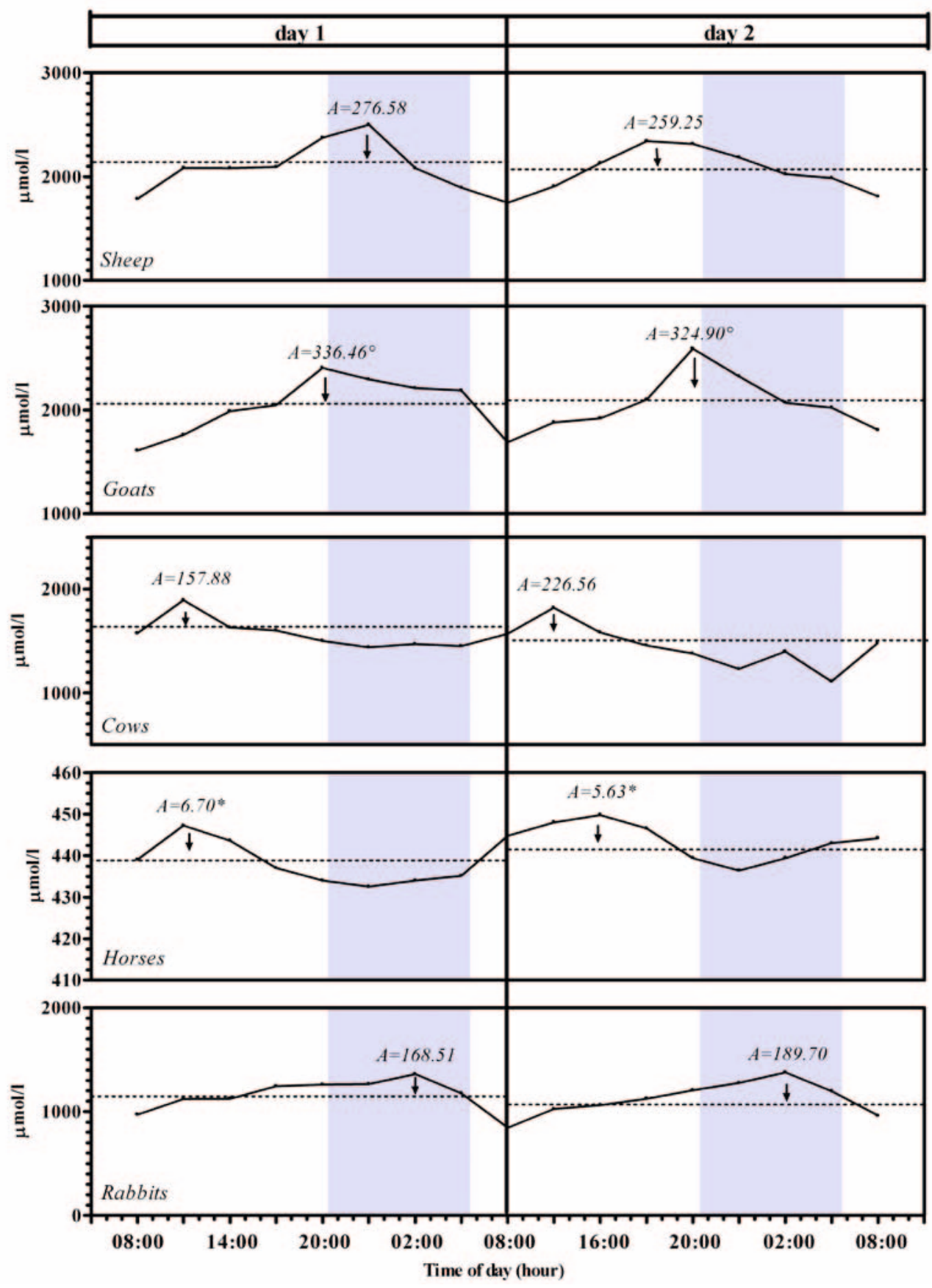

Figure 2. Daily patterns of Oxy-ads observed in sheep, goats, cows, horses and rabbits during the 48 hours of monitoring. Grey bars indicate the dark phase of photoperiod. Broken line indicates mesor value. A indicates amplitude value.

* Statistically significant as compared with other species

- Statistically significant as compared with cows and rabits

of hydroperoxides, Oxy-ads and $\mathrm{SHp}$ in the five species studied during the $48 \mathrm{~h}$ of monitoring are shown in Figures 1-3.

Hydroperoxides showed daily rhythmicity in all species studied. Amplitude of rhythm was higher in rabbits (statistically significant) than in other species
(Figure 1). The hydroperoxide rhythmicity was more robust in goats and horses (statistically significant) than cows and rabbits (Figure 4).

Oxy-ads showed daily rhythmicity in all species studied. The amplitude of rhythm was lower in horses (statistically significant) than the other species (Figure 2), 


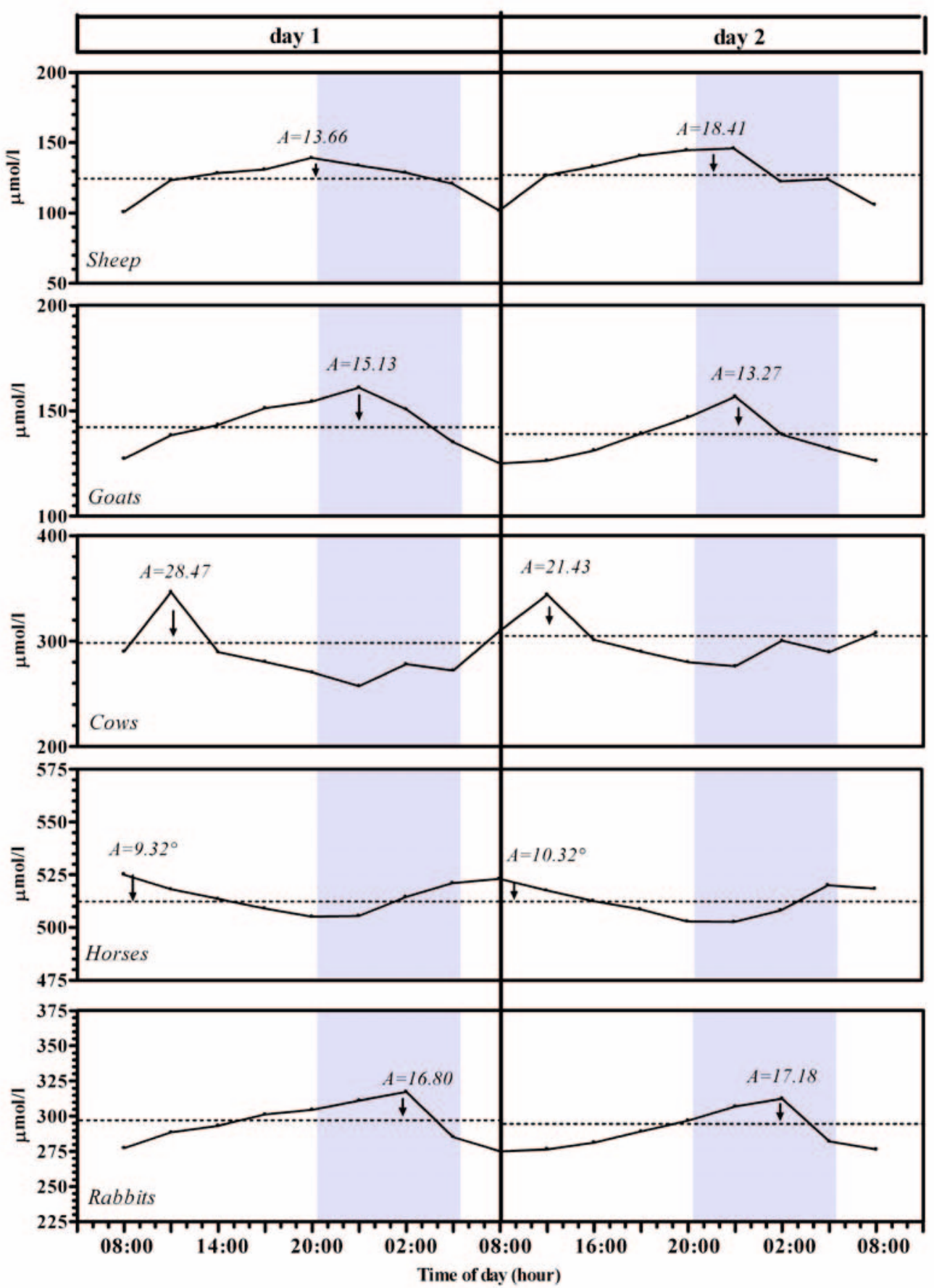

Figure 3. Daily patterns of SHp observed in sheep, goats, cows, horses and rabbits during the 48 hours of monitoring. Grey bars indicate the dark phase of photoperiod. Broken line indicates mesor value. A indicates amplitude value.

- Statistically significant as compared with cows

and higher in goats (statistically significant) than cows and rabbits (Figure 2). The Oxy-ads rhythmicity was less robust in cows (statistically significant) than in goats, sheep and horses (Figure 4).

$\mathrm{SHp}$ showed daily rhythmicity in all species studied. The amplitude of rhythm was lower in horses (statistically significant) than in cows (Figure 3). The SHp rhythmicity was less robust in cows (statistically significant) than other species (Figure 4) and higher in horses (statistically significant) than goats and sheep (Figure 4).

In all studied parameters, diurnal acrophases were observed in cows and horses. They were statistically 


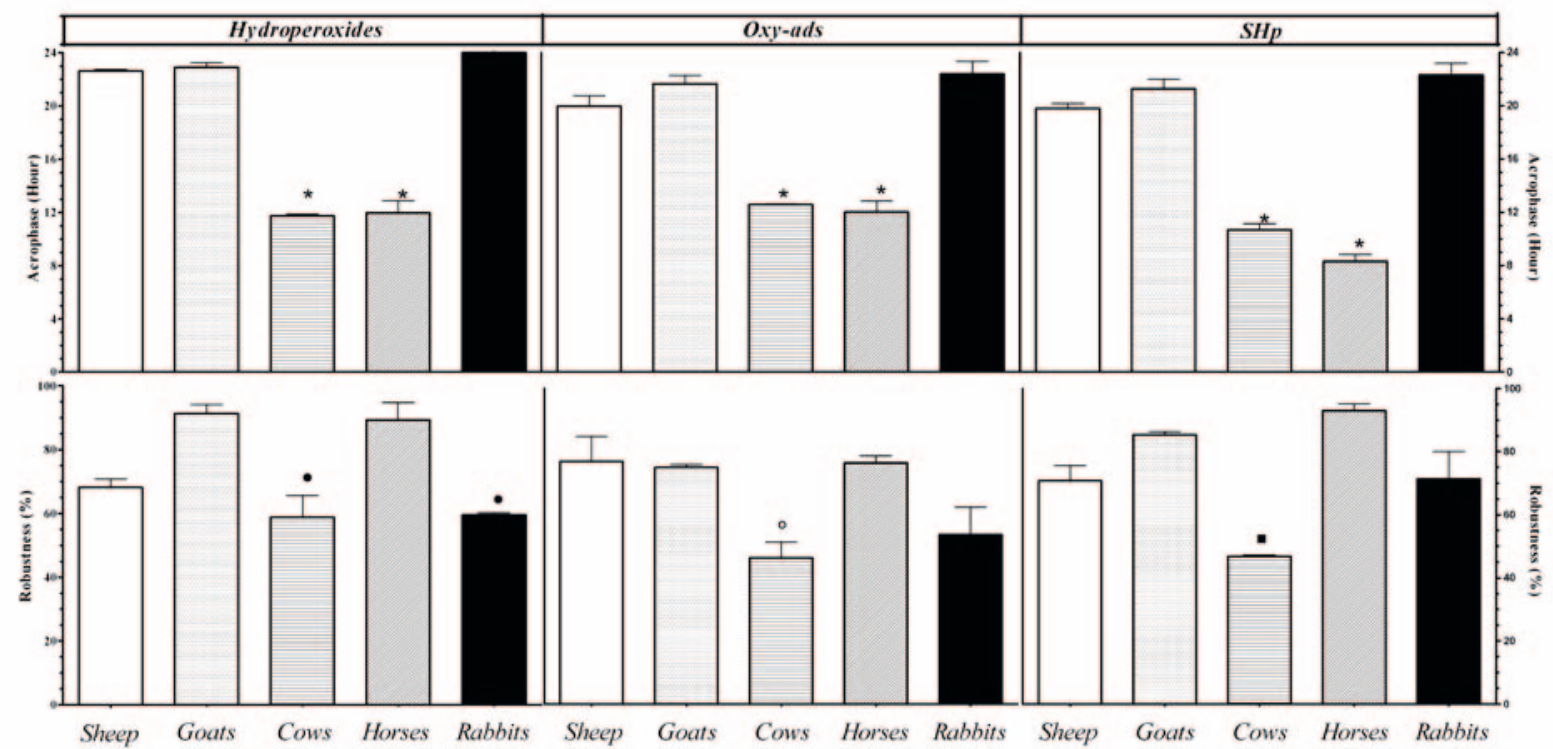

Figure 4. Acrophase and robustness of rhythm observed in sheep, goats, cows, horses and rabbits during the 48 hours of monitoring. Data are expressed as mean \pm SD

Significance: * vs. sheep, goats, rabbit $P<0.01$

${ }^{\circ} v s$ s sheep, goats and horses $P<0.05$

-vs. goats and horse $P<0.05$

- vs. other $P<0.05$

different (statistically significant) from the nocturnal acrophases observed in sheep, goats and rabbits (Figure 4).

\section{Discussion}

Generation of ROS takes place continually in a fluctuating way driven by a circadian clock. Healthy cells also homeostatically oppose ROS through the use of naturally present antioxidant showing daily oscillation.

Our results showed daily variations of hydroperoxides, Oxy-ads and SHp in all studied species. Species-specificity in these variations, with differences in the amount of hydroperoxides, oxy-ads and SHp concentrations, amplitude, acrophase and robustness among the species studied were observed.

Considering that in the 48 hours of monitoring goats showed the lowest hydroperoxide serum concentration the lowest response of serum barrier to oxidation would be expected. Therefore, low serum concentrations were observed only for SHp and not for Oxy-ads. In goat, this parameter showed the highest value. Compared to goats, in horses the opposite trend of oxidant and antioxidants were observed. Hydroperoxide and SHp showed high serum concentrations, and Oxy-ads showed low serum concentration. The same Oxy-ads and SHp serum concentrations in sheep and goats were observed, but these two species did not show the same hydroperoxides serum concentration.

Completely different trends were observed in rabbits that showed the highest hydroperoxide serum concentration and Oxy-ads and SHp values similar to the other species.

Species-specificity of hydroperoxide and serum barrier to oxidation was observed to a lesser extent than their rhythmicity. The amplitude is half the range of oscillation. It identifies the boundaries of the oscillation. Therefore, real circadian rhythms are not necessarily symmetrical, so that the amplitude below the mean level may be different from the amplitude above the mean level. As observed for the mean level, different amplitude values were observed between hydroperoxide production and the antioxidant barrier in each species. Rabbits showed the highest amplitude of hydroperoxide rhythm, but not the highest amplitude of serum antioxidant barrier. Horses showed the lowest Oxy-ads amplitude values, but not the lowest hydroperoxide; $\mathrm{SHp}$ amplitude values were lower only than cows. The robustness of a rhythm is distinct from its amplitude, as well as from its other parameters. Robustness measures the stability of a rhythm. The higher the robustness value the more probability that the daily oscillation is similar from one day to another, and the result is a rhythm 
quite consistent (almost stationary). On the contrary, a low robustness value indicates a rhythm rather variable (hardly stationary). There is a reciprocal relationship between the robustness of the endogenous circadian timing system and its dependency on regularly timed synchronisers [22]. On the basis of this knowledge, the low robustness value of hydroperoxides and serum barrier to oxidation observed in cows showed a low stability of oxidant and antioxidant metabolism in this species. On the contrary, a quite consistent rhythm of hydroperoxides, Oxy-ads and SHp was observed in the other species studied.

Interestingly, for all parameters studied, the acrophase of rhythm (the time at which the peak of a rhythm occurs) showed similar aspect between sheep, goats and rabbits, that showed nocturnal acrophases and between cows and horses that showed diurnal acrophases. In sheep, goats and rabbits the acrophase of hydroperoxides was preceded of about two hours by

\section{References}

[1] Križanović D., Sušić V., Božić P., Štoković I., Ekert-Kabalin A., Changes of bovine blood lipid peroxides and some antioxidants in the course of growth, Veterinarski Arhiv, 2008, 78, 269-278

[2] Kirschink N., de Moffarts B., Lekeux P., The oxidant/antioxidant equilibrium in horses, Vet. J., 2008, 177, 178-191

[3] Lykkesfeldt J., Svendsen O., Oxidants and antioxidants in disease: Oxidative stress in farm animals, Vet. J., 2007, 173, 502-511

[4] Bergero D., Miraglia N., Schimone A., Polidori M., Prola L., Effect of dietary polyunsaturated fatty acids and vitamin $\mathrm{E}$ on serum oxidative status in horses performing very light exercise, Ital. J. Anim. Sci., 2004, 3, 141-145

[5] Piccione G., Fazio F., Giannetto C., Assenza A., Caola G., Oxidative stress in thoroughbreds during official 1800-meter races, Veterinarski Arhiv, 2007, 77, 219-227

[6] Piccione G., Borruso M., Fazio F., Grasso F., Caola G., Oxidative stress evaluation during milking period in the ewes, J. Appl. Anim. Res., 2006, 29, 109-112

[7] Piccione G., Casella S., Assenza A., Fazio F., Caola G., Evaluation of serum homocysteine and oxidative stress during lactation in ewes, Czech J. Anim. Sci., 2008, 53, 462-465

[8] Di Trana A., Celi P., Claps S., Fedele V., Rubino R., The effect of hot season and nutrition on the oxidative status and metabolic profile in dairy goats during mild lactation, Anim. Sci., 2006, 82, 717-722 the acrophase of oxy-ads and SHp that were coincident. In cows and horses the acrophase of hydroperoxides was preceded by the acrophase of $\mathrm{SHp}$ and followed by the acrophase of Oxy-ads.

Therefore, being able to exclude the effect of environmental conditions [23], such as light-dark cycle, and of feeding, on the studied parameters, as all studied species were subjected to the same experimental protocol, we can claim that, in herbivorous there is a species-specific daily rhythm of hydroperoxide production and a speciesspecific response of serum barrier to oxidation. Even thought, in each studied species, different mesor and amplitude were observed between hydroperoxides and serum barrier to oxidation, a synergism of rhythmicity between hydroperoxide production and the response of serum barrier to oxidation, underlined by synergism in acrophases and robustness of rhythm, was observed.

[9] Pasquini A., Luchetti E., Marchetti V., Cardini G., Iorio E., Analytical performances of dROMs test and BAP test in canine plasma. Definition of the normal range in healthy Labrador dogs, Vet. Res. Commun., 2008, 32, 137-143

[10] Pedernera-Romano M., Salvin H., Celi P., Garcia S.C., Horadagoda A., Barchia I., et al., Oxidative stress in early lactating cows fed to produce 6000 or 9000 milk/lactation in pasture-based system: preliminary results, Proceeding of the Dairy Research Foundation Symposium (Camden, Australia), 2006, 150-152

[11] Piccione G., Borruso M., Giannetto C., Morgante M., Giudice E., Assessment of oxidative stress in dry and lactating cows, Acta Agric. Scand. A Anim. Sci., 2007, 57, 101-104

[12] Shadan F.F., Sleep-wake cycle, aging and cancer, J. Appl. Biomed., 2008, 6, 131-138

[13] Hardeland R., Coto-Montes A., Poeggeler B., Circadian rhythms, oxidative stress, and oxidative defence mechanisms, Chronobiol. Int., 2003, 20, 921-962

[14] Giannetto C., Fazio F., Assenza A., Caola G., Pennisi P., Piccione G., Circadian rhythms of redox states and total locomotor activity in dairy cattle, Czech J. Anim. Sci., 2010, 55, 183-189

[15] Piccione G., Giannetto C., Fazio F., Pennisi P., Caola G., Evaluation of total locomotor activity and oxidative markers daily rhythms in sheep, Biol. Rhythm Res., 2010, in press, DOI 10.1080/09291010903408225 
[16] Piccione G. Giannetto C., Fazio F., Assenza A., Caola G., Influence of reproductive status on the daily rhythms of oxidative stress markers in Ovis aries, Cent. Eur. J. Biol., 2010, 5, 384-390

[17] Franzini A., Luraschi P., Pagani A., Direct measurement of reactive oxygen metabolites in blood serum: analytical assessment of a novel method, Biochimica Clinica, 1996, 5-6, 89

[18] Alberti A., The d-ROms test. Model and chemical basis, Proceeding I.Co.C.E.A. National Council of Research (Bologna, Italy), 1997

[19] lamele L., Fiocchi R., Vernocchi A., Evaluation of an automated spectrophotometric assay for reactive oxygen metabolites in serum, Clin. Chem. Lab. Med., 2002, 40, 673-676
[20] Nelson K., Tong J.L., Lee J.K., Halberg F., Methods for cosinor rhythmometry. Chronobiologia, 1979, 6, 305-323

[21] Refinetti R., Non-stationary time series and the robustness of circadian rhythms, J. Theor. Biol., 2004, 227, 571-581

[22] Berger J., Advances in chronohaematology, Appl. Biomed., 2008, 6, 65-72

[23] Dardante H., Does a Melatonin-dependent circadian oscillator in the pars tuberalis drive prolactin seasonal rhythmicity?, J. Neuroendocrinol., 2007, 19, 657-666 